\title{
Análise de agrupamento de diferentes densidades de marcadores no mapeamento genético por varredura genômica ${ }^{1}$
}

\author{
Marcelo Jangarelli ${ }^{2}$ Ricardo Frederico Euclydes ${ }^{3}$, Cosme Damião Cruz ${ }^{4}$, Paulo Roberto Cecon ${ }^{5}$, \\ Antonio Policarpo Souza Carneiro ${ }^{6}$
}

\section{RESUMO}

A simulação tem contribuído para o avanço da genômica nas diversas áreas do melhoramento genético. Foram simulados mapeamentos genéticos utilizando diferentes densidades de marcadores para estimar os valores fenotípicos na seleção assistida por marcadores (SAM), em características quantitativas com valores de herdabilidade de 0,10; 0,40; e 0,70. Procedeu-se a análise de agrupamento com os desempenhos fenotípicos, cuja finalidade foi obter estruturas de classificação entre as densidades visando à otimização na detecção de QTL. O sistema de simulação genética (Genesys) foi utilizado para três genomas (cada qual constituído de uma única característica cuja distinção estava no valor da herdabilidade) e para as populações base e inicial. Cada população inicial foi submetida à seleção assistida por marcadores por 20 gerações consecutivas, em que os genitores selecionados acasalavam-se seletivamente entre os melhores e os piores. O mapeamento empregando de média a alta densidade de marcadores assinalou eficiência nos progressos fenotípicos obtidos com a SAM. Menores quantidades de marcadores são requeridas para manter determinado poder de detecção de QTL à medida que se eleva a magnitude da herdabilidade. A análise de agrupamento indicou otimização e correspondência nos incrementos fenotípicos ao admitir as densidades de 4 e 6 cM; 4, 6, 8 e 10 cM; e 6 e 8 cM para as herdabilidades de 0,10; 0,40; e 0,70, respectivamente.

Palavras-chave: Análise de cluster, herdabilidade, seleção genômica, simulação, QTL.

\section{ABSTRACT}

\section{Cluster analysis of different marker densities in genetic mapping using genome scan}

Simulation has contributed to the advancement of genomics in the different areas of genetic improvement. Genetic mappings were simulated using different densities of genetic markers to estimate phenotypic values of quantitative traits with heritabilities of 0.10 ; 0.40 and 0.70 in marker assisted selection (MAS). Cluster analysis with phenotypic performances was carried out to generate classification structures among the densities aiming to optimize QTL detection . The genetic simulation system (Genesys) was used to simulate three genomes (each consisting of a single characteristic differing in the heritability value) and the base and original populations. Each initial population was subjected to selection assisted by markers for 20 consecutive generations, in which selected parents mated selectively, between best and worst. The mapping using medium to high marker density showed efficiency in the phenotypic progress

\footnotetext{
Recebido para publicação em julho de 2009 e aprovado em novembro de 2010

${ }^{1}$ Trabalho extraído da tese de doutorado do primeiro autor. Financiado pelo CNPq.

2 Zootecnista, Doutor. Universidade Federal Rural do Rio de Janeiro gmejanga@hotmail.com

Zootecnista, Ph. Doctor. Universidade Federal de Viçosa, Departamento de Zootecnia. Av. P. H. Rolfs, s/n, 36570-000, Viçosa, MG, Brasil. rbaja@ufv.br

${ }^{4}$ Engenheiro-Agrônomo, Doutor. Universidade Federal de Viçosa, Departamento de Biologia Geral, Av. P. H. Rolfs, s/n, 36570-000 Viçosa, MG, Brasil. cdcruz@ufv.br

${ }^{5}$ Engenheiro-Agrônomo, Doutor. Universidade Federal de Viçosa, Departamento de Informática (Área Estatística), Av. P. H. Rolfs, s/n, 36570-000 Viçosa, MG, Brasil. cecon@dpi.ufv.br

${ }^{6}$ Zootecnista, Doutor. Universidade Federal de Viçosa, Departamento de Informática (Área Estatística), Av. P. H. Rolfs, s/n, 36570-000 Viçosa, MG, Brasil. policarpo@dpi.ufv.br
} 
obtained with MAS. Smaller marker quantities are required to maintain power of QTL detection with increase in heritability. The cluster analysis indicated optimization and correspondence in phenotypic increases, when allowing the densities of 4 and $6 \mathrm{cM}, 4,6,8$ and $10 \mathrm{cM}$, and 6 and $8 \mathrm{cM}$ for the heritabilities of $0.10 ; 0.40$ and 0.70 , respectively.

Key words: Cluster analysis, heritability, genome selection, simulation, QTL.

\section{INTRODUÇÃO}

O melhoramento genético, por meio da seleção e de cruzamentos, objetiva a cada geração aumentar a frequência de genes associados às características fenotípicas favoráveis, otimizando a produtividade. O avanço no conhecimento de técnicas moleculares permite a identificação e caracterização de regiões genômicas associadas às características quantitativas (QTL Quantitative Trait loci), implementando os métodos tradicionais de melhoramento por meio da seleção assistida por marcadores (SAM), maximizando os ganhos genéticos (Ledur \& Schmit, 2000).

A deteç̧ão de QTL na SAM pode ser realizada por meio da varredura genômica (Vieira et al., 2006). Esse método consiste em utilizar marcadores moleculares posicionados ao longo de todo o genoma ou de cromossomos individuais a fim de identificar regiões que afetam características quantitativas de interesse. O intervalo na disposição dos marcadores no genoma é um indicativo da resolução do mapeamento. Quanto menor for a distância entre marcadores adjacentes utilizados na análise de ligação maior será a resolução do mapeamento. Essa alta resolução permite a construção de mapas genéticos altamente densos, favorecendo o mapeamento e a estimação da magnitude dos efeitos dos QTL (Ledur et al., 2004; Ambo et al., 2008; Rodrigues et al., 2010).

Dessa forma, os marcadores moleculares, quando suficientemente numerosos, contribuem para uma cobertura adequada do genoma. Entretanto, em baixa densidade eles tornam-se fatores limitantes em estudos de análise de QTL, pois ao utilizar poucos marcadores diminuem as chances de encontrar desequilíbrio de ligação entre marcador e QTL. Esse fato é agravado quando os marcadores são distribuídos aleatoriamente em vez de serem estrategicamente dispostos ao longo do genoma.

Na tentativa de identificar densidades de marcadores similares em estudos de detecção de QTL pode-se empregar a análise de agrupamento (Cluster Analysis). Ela é uma técnica de estatística multivariada utilizada para classificar elementos ou medidas em categorias similares (Ferreira, 2008). As mensurações provenientes da estimação de parâmetros genéticos e fenotípicos poderão ser utilizadas como medidas de variáveis classificatórias, propiciando o agrupamento de densidades (número de marcadores) que resultem em estimativas semelhantes, de acordo com os valores assumidos pelo parâmetro considerado (Malone et al., 2007).

Objetivou-se com este trabalho avaliar a eficiência de diferentes densidades de marcadores moleculares na resposta à seleção assistida por marcadores, via processo de simulação, aplicando técnicas de agrupamento para identificação de densidades similares na varredura genômica.

\section{MATERIAL E MÉTODOS}

Foram utilizados neste trabalho dados simulados pelo sistema de simulação genética Genesys (Genetic System), versão 2009 (Euclydes, 2009). Esse sistema foi escrito na linguagem de programação FORTRAN. Ele foi desenvolvido para permitir a simulação de genomas complexos, capacitando o estudo de pressuposições genéticas e estatísticas de interesse nos processos de seleção, entre outras possibilidades.

Foram simulados três genomas hipotéticos, separadamente, cuja distinção estava no valor da herdabilidade da característica. Cada genoma foi constituído de uma característica quantitativa com herdabilidade de 0,10; 0,40; e 0,70 , respectivamente.

Cada genoma proposto apresentou 958 centiMorgan (cM) de extensão; os marcadores moleculares foram dispostos estrategicamente, em quantidade variável de acordo com a densidade desejada no mapeamento; ficou constituído por 200 locos quantitativos (QTL), associados à característica, distribuídos ao longo de 40 cromossomos de tamanho aleatório; os efeitos aditivos dos QTL foram simulados seguindo a distribuição normal dos dados fenotípicos; os locos quantitativos foram bialélicos e não possuíam desvios de dominância e nem epistasia; as frequências gênicas iniciais foram iguais para ambos os sexos; as frequências gênicas iniciais para os marcadores moleculares seguiram distribuição normal, apresentando valores próximos a 0,5 ; os efeitos de ambiente foram simulados conforme a distribuição normal; os dados fenotípicos simulados apresentaram média de 10 unidades e desvio-padrão de duas unidades.

Para cada estrutura genômica simulada foi construída uma população base composta de 500 machos e 500 fêmeas (1.000 indivíduos), todos heterozigotos, não aparenta- 
dos entre si. Para a obtenção das populações iniciais, 50 machos e 50 fêmeas foram escolhidos aleatoriamente em cada população base. Cada cruzamento (uma fêmea/macho) produziu 10 filhos, resultando 500 descendentes que formavam as populações iniciais (500 indivíduos). Cada população inicial foi submetida à seleção assistida por marcadores (SAM) por 20 gerações consecutivas com 20 repetições, visando minimizar os efeitos da oscilação genética. A seleção foi conduzida com a finalidade de incrementar o valor fenotípico.

A partir de cada população inicial, os reprodutores foram selecionados com base em seus genótipos, de acordo com o número de marcadores moleculares identificados que estariam estatisticamente associados aos locos quantitativos. Dessa forma, os genitores eleitos em cada geração representavam os indivíduos detentores de maior informatividade dos marcadores, ou seja, aqueles que apresentavam maior número de marcadores ligados aos QTL. A cada geração, os 50 machos e as 50 fêmeas (uma fêmea/macho) que obtiveram o maior número de marcadores informativos foram acasalados. O número de progênies em cada cruzamento foi igual a 10. Esses descendentes formaram a geração seguinte. Na seleção assistida por marcadores foi adotada a estratégia de genotipagem seletiva, acasalando os melhores machos com as piores fêmeas, e vice versa, entre os 100 genitores selecionados (50 machos e 50 fêmeas). Essa estratégia foi escolhida face à sua eficiência em aumentar o poder de detecção de QTL para a característica sobre a qual os indivíduos foram selecionados, maximizando a variabilidade genética na amostra, segundo afirmam Ruy et al. (2005) e Rosa (2007). Ela otimiza a variabilidade genética entre os genitores selecionados, favorecendo o mapeamento de QTL por meio da utilização racional dos animais (Carneiro et al., 2007; Neves et al., 2009). Entre outras vantagens, esse acasalamento entre indivíduos divergentes favorece a segregação de alelos e proporciona maior poder nas análises estatísticas, devido à alta heterozigosidade nos QTL, além das fases de ligação entre marcadores e de os QTL serem mais consistentes. Por sua vez, este acasalamento estratégico aumenta o número de marcadores e de cruzamentos informativos (Silva et al., 2005; Peixoto et al., 2009).

A seleção assistida por marcadores foi conduzida em diferentes densidades de marcadores. Elas foram representadas pela distância, em centiMorgan (cM), entre marcadores adjacentes. Foram admitidas 15 densidades: 2, 4, 6, 8, 10, 12, 14, 16, 18, 20, 22, 24, 26, 28 e $30 \mathrm{cM}$, correspondendo à utilização de 479, 239, 159, 119, 95, 79, 68, 59, 53, 47, 43, 39, 36, 34 e 31 marcadores moleculares. Dessa forma, obtida a população inicial para cada genoma (nível de herdabilidade), foram praticadas 15 seleções assistidas por marcadores.
A identificação de associações entre marcadores e QTL na SAM foi obtida por meio do método da marca simples. Esse método verifica a associação entre cada marcador e a característica de interesse. Utilizou-se a análise de regressão linear entre os genótipos dos marcadores flanqueadores do QTL e os valores fenotípicos dos descendentes dos acasalamentos. Foi adotado o nível de significância genômica de 5\% na regressão linear simples.

Para comparação das diferentes densidades admitidas na SAM foram estimados os valores fenotípicos médios em cada geração. Com o propósito de buscar uma estrutura classificatória entre as densidades, foi aplicada a análise estatística multivariada de agrupamento. Na aplicação dessa análise, as densidades foram consideradas como tratamentos. As densidades de 2, 4, 6, 8, 10, 12, 14, $16,18,20,22,24,26,28$ e $30 \mathrm{cM}$ representaram os tratamentos 1, 2, 3, 4, 5, 6, 7, 8, 9, 10, 11, 12, 13, 14 e 15, respectivamente. Cada geração representou uma variável, totalizando 20. Procedeu-se a análise utilizando os valores fenotípicos médios obtidos ao longo das 20 gerações.

Visando minimizar os problemas de colinearidade entre as variáveis (gerações), os valores fenotípicos estimados foram padronizados e então utilizados dois métodos de agrupamento: método de otimização de Tocher e método da ligação completa, na tentativa de ambos corroborarem na proposta de formação de grupos com estrutura classificatória similar entre as densidades. Em ambos os métodos a distância euclidiana média foi utilizada para quantificar as dissimilaridades entre os tratamentos (densidades de marcadores), visto que as estimativas dos valores fenotípicos representaram um único valor médio sintetizando as repetições. Além disso, essa medida não é influenciada pelo número de variáveis consideradas, sendo bastante difundida em programas de melhoramento genético (Nascimento Filho et al., 2001).

As análises multivariadas foram realizadas em cada nível de herdabilidade $(0,10 ; 0,40$; e 0,70$)$. Elas foram processadas utilizando o Sistema para Análises Estatísticas e Genéticas - SAEG, descrito por Ribeiro Junior \& Melo (2008).

\section{RESULTADOS E DISCUSSÃO}

\section{Característica de baixa herdabilidade $\left(h^{2}=0,10\right)$}

Na Tabela 1 são apresentados os valores fenotípicos médios e seus respectivos desvios-padrão obtidos ao longo de 20 gerações sob SAM. Foram admitidas diferentes resoluções no mapeamento, que se distinguiram pela distância (centiMorgan - cM) entre marcadores adjacentes. Essa disposição ficou fixa, possibilitando uma varredura genômica em distintas quantidades de marcadores.

Todos os processos de seleção iniciaram-se no mesmo valor fenotípico na população experimental (10 unida- 


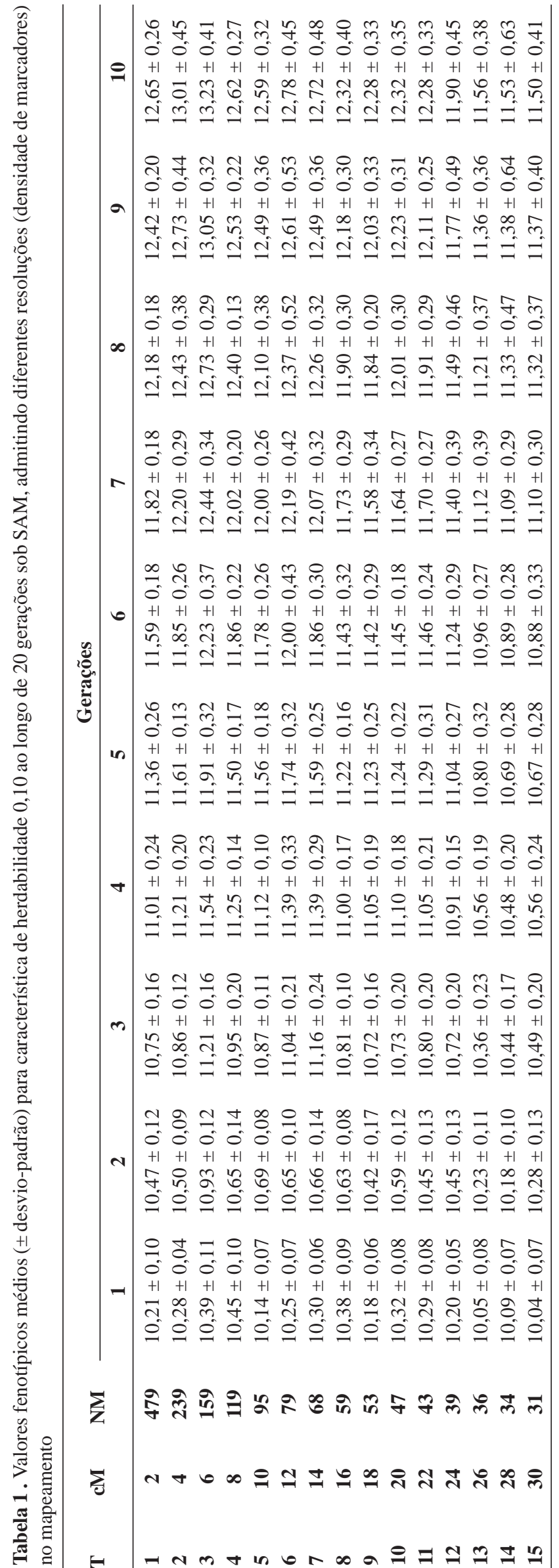

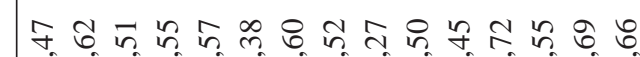

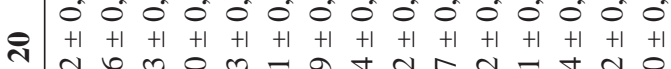
กิ

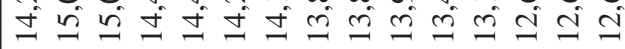

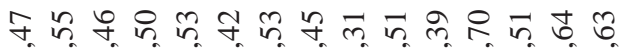
$\Omega\left(\begin{array}{llllllll}0 & 0\end{array}\right.$

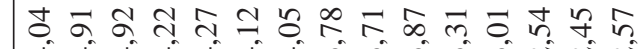

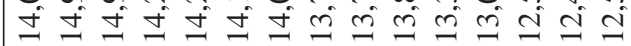

ๆ

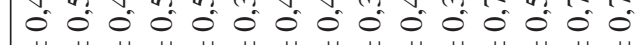

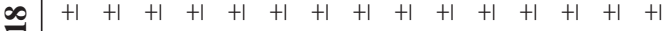

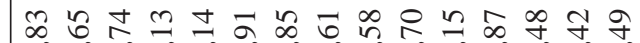

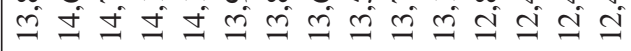

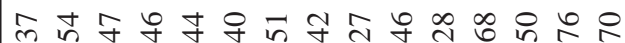

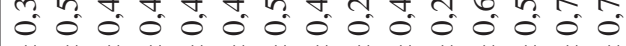

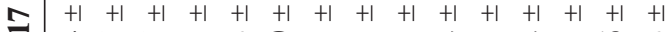

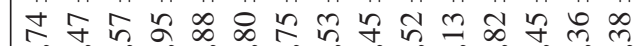

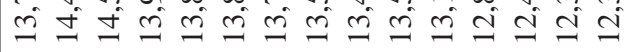

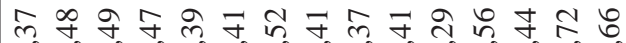
000

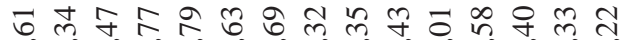

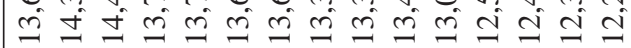

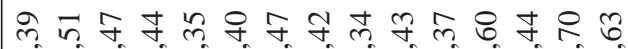

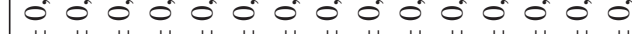
L

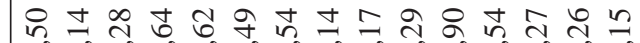

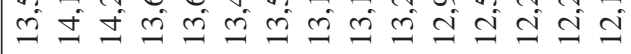

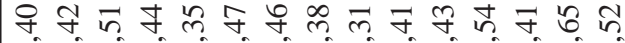

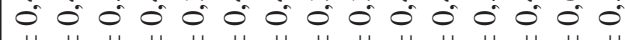

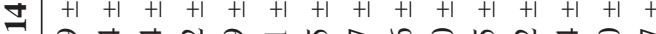

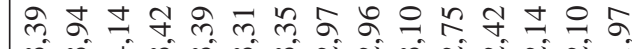

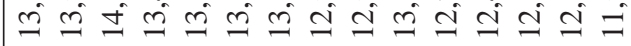

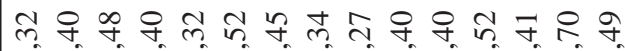

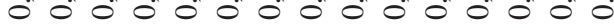

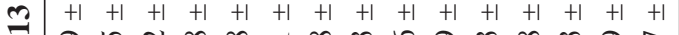
근

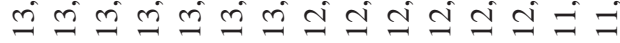

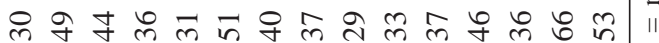

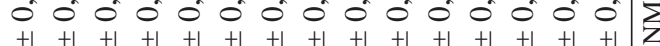

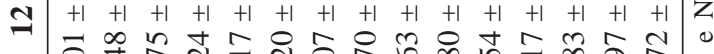
ले ले

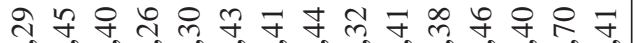

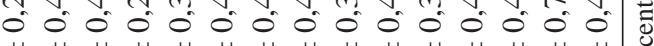

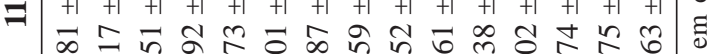
สิต ติ

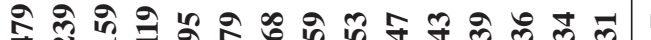
ব

$\sum$ ก 
des), possibilitando comparações entre as densidades. Logo nas primeiras gerações foram observados padrões de similaridade entre os mapeamentos que adotaram alta resolução (maior número de marcadores), estendendo-se até a $20^{a}$ geração. Segundo Peixoto et al. (2009), quanto mais próximo o QTL estiver do marcador maior será o poder de detecção do mapeamento. As densidades de 4 e 6 cM apresentaram desempenho superior, principalmente a partir das gerações intermediárias. Essa superioridade tornou-se de maior magnitude com as subsequentes gerações sob SAM. Resultados expressivos também foram detectados nas seleções que utilizaram até 68 marcadores moleculares (MM). Todavia, os mapas de baixa densidade (26, 28 e $30 \mathrm{cM}$ ) proporcionaram os piores resultados ao longo de todas as gerações, face ao seu baixo poder de detecção. Neles, a posição do QTL não poderá ser precisamente determinada, em virtude do confundimento do seu efeito e da sua posição.

Apesar do aumento no número de estudos relacionados ao mapeamento de QTL, a resolução com que eles são conduzidos ainda é considerada baixa, em geral, estando na ordem de 20 a 30 cM (Ledur et al., 2003). Estudos de simulação desenvolvidos por Moreau et al. (1998) evidenciaram que $5 \mathrm{cM}$ seria uma distância adequada entre os marcadores que contêm um QTL para uso na SAM, corroborando com os resultados deste trabalho. Também por simulação Darvasi et al. (1993) realizaram trabalhos envolvendo o espaçamento entre marcadores adjacentes, constatando que o poder de detecção de QTL foi semelhante ao usar marcadores espaçados a uma distância igual ou inferior a $10 \mathrm{cM}$, enquanto decréscimos no poder de detecção de QTL foram observados ao admitir espaçamento igual ou superior a $20 \mathrm{cM}$. Os resultados da análise de agrupamento para o poder de deteç̧ão de QTL, analisados indiretamente pelo incremento fenotípico na SAM, foram mais discriminantes, evidenciando densidades similares ao se admitir distâncias entre marcadores inferiores a $10 \mathrm{cM}$ ou superiores a $20 \mathrm{cM}$. Utilizar muitos marcadores estrategicamente espaçados maximiza a probabilidade de se identificar ligações entre marcadores e QTL, otimizando o ganho fenotípico na SAM, conforme relatado por Hillel (1997).

A Tabela 2 indicou a formação de cinco grupos distintos pelo método de Tocher. No grupo 1 foram classi-

Tabela 2 . Grupos, limite e distâncias obtidas entre os tratamentos (densidades de marcadores) pela aplicação do método de Tocher para herdabilidade de 0,10

\begin{tabular}{lcccc}
\hline Número do grupo & Limite $(\grave{e})$ & $\begin{array}{c}\text { Distância } \\
\text { obtida }\end{array}$ & $\begin{array}{c}\text { Número de } \\
\text { tratamentos }\end{array}$ & $\begin{array}{c}\text { Tratamentos } \\
\text { pertencentes* }\end{array}$ \\
\hline $\mathbf{1}$ & 0,2562 & 0,07 & 2 & $13 ; 14$ \\
$\mathbf{1}$ & 0,2562 & 0,10 & 3 & $13 ; 14 ; 15$ \\
$\mathbf{2}$ & 0,2562 & 0,08 & 2 & $8 ; 10$ \\
$\mathbf{2}$ & 0,2562 & 0,11 & 3 & $8 ; 10 ; 9$ \\
$\mathbf{3}$ & 0,2562 & 0,09 & 2 & $6 ; 7$ \\
$\mathbf{3}$ & 0,2562 & 0,14 & 3 & $6 ; 7 ; 4$ \\
$\mathbf{3}$ & 0,2562 & 0,15 & 4 & $6 ; 7 ; 4 ; 1$ \\
$\mathbf{3}$ & 0,2562 & 0,14 & 5 & $6 ; 7 ; 4 ; 1 ; 5$ \\
$\mathbf{4}$ & 0,2562 & 0,13 & 2 & $11 ; 12$ \\
$\mathbf{5}$ & 0,2562 & 0,25 & 2 & $2 ; 3$ \\
\hline
\end{tabular}

* Tratamentos: 1 (2 cM); 2 (4 cM); 3 (6 cM); 4 (8 cM); 5 (10 cM); 6 (12 cM); 7 (14 cM); 8 (16 cM); 9 (18 cM); 10 (20 cM); 11 (22 cM); 12 (24 cM); 13 (26 cM); 14 (28 cM); e 15 (30 cM).

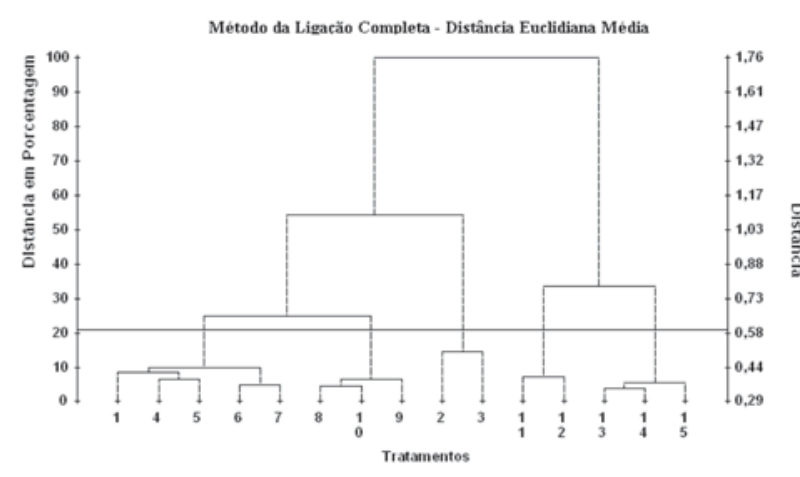

Figura 1 . Dendrograma do método da ligação completa pela análise de 15 tratamentos (densidades de marcadores) para herdabilidade de 0,10 .

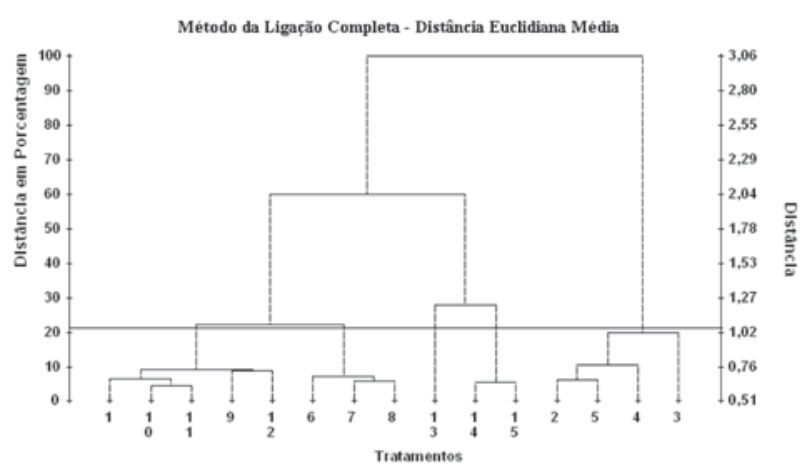

Figura 2 . Dendrograma do método da ligação completa pela análise de 15 tratamentos (densidades de marcadores) para herdabilidade de 0,40

Rev. Ceres, Viçosa, v. 57, n.6, p. 695-700, nov/dez, 2010 


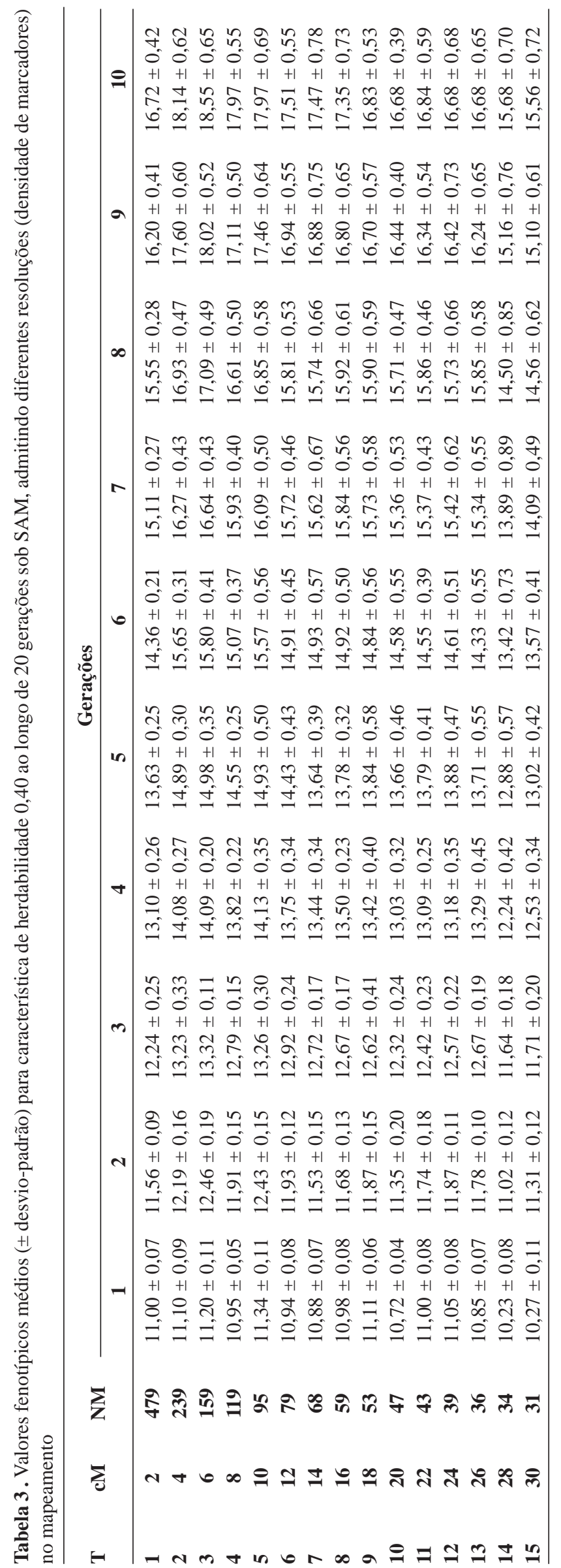

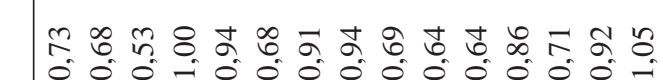
ล $+1+1+1+1+1+1+1+1+1+1+1+1+1+1+1$

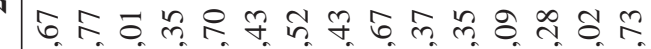
ถิ

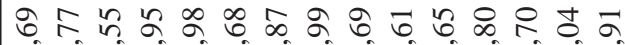

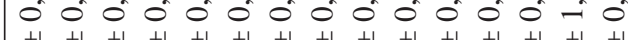

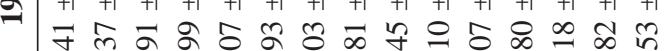

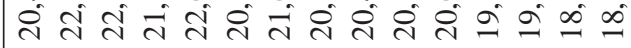

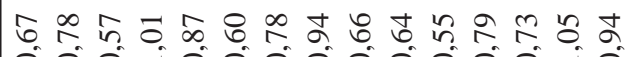

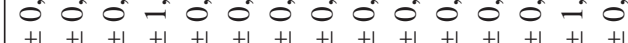

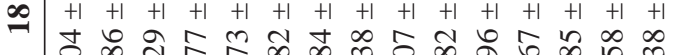
กิ

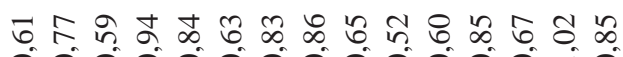

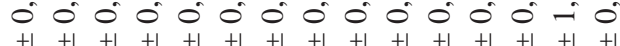

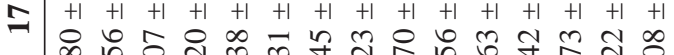
जे הิ สิ

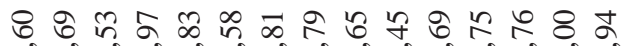

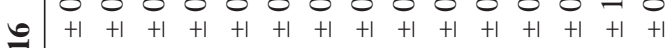

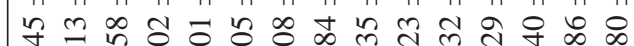
बे ते

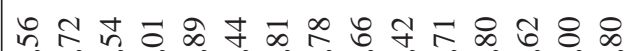
o co ô $ص+1+1+1+1+1+1+1+1+1+1+1+1+1+1+1$

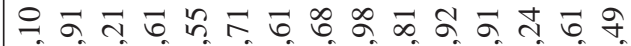

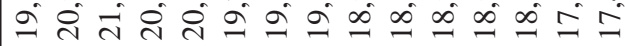

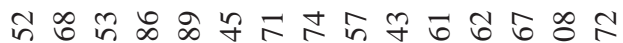

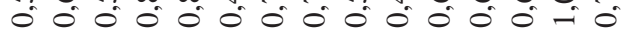
$\Xi+1+1+1+1+1+1+1+1+1+1+1+1+1+1+1$ ㄲ ๆ

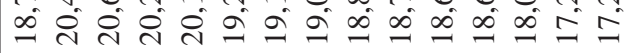

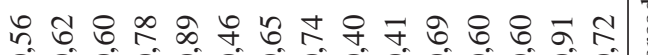

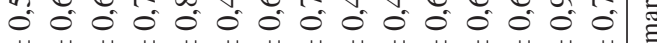

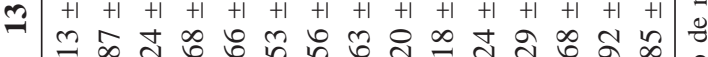

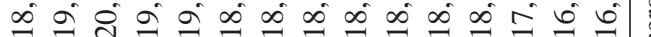

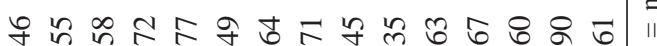

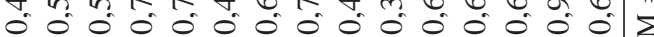
$\simeq+1+1+1+1+1+1+1+1+1+1+1+1+1+1+1\}$

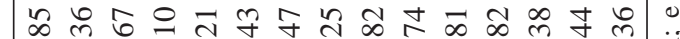

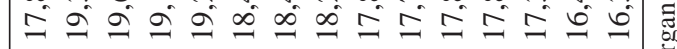

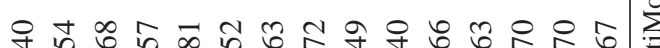
ô

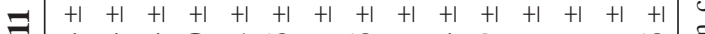
茫

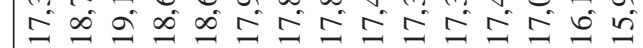

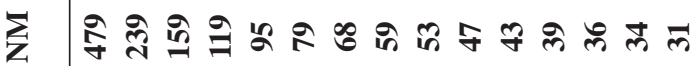

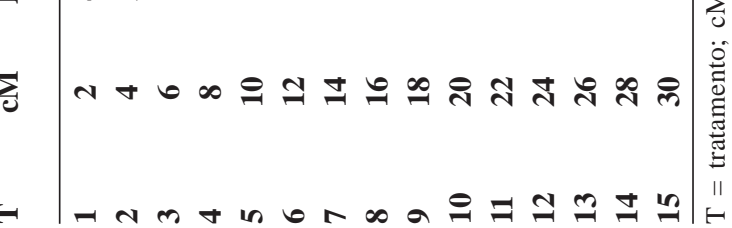

Rev. Ceres, Viçosa, v. 57, n.6, p. 695-700, nov/dez, 2010 
ficados os espaçamentos de 26, 28 e 30 cM. O grupo 2 de 16,18 e 20 cM. O terceiro grupo foi formado pelas densidades de 2, 8, 10, 12 e 14 cM. As distâncias de 22 e 24 cM agruparam-se no quarto grupo. Já o grupo 5 compreendeu as saturações com um marcador a cada 4 e 6 cM. Evidenciou-se, com base nos incrementos fenotípicos médios, a inferioridade do grupo 1 e a superioridade do grupo 5. Ganhos fenotípicos expressivos também foram obtidos pelas densidades que constituíram o grupo 3.

O dendrograma estabelecido pelo método da ligação completa está apresentado na Figura 1. Seu resultado diverge com relação ao método de Tocher por não estabelecer um número ótimo de grupos, ilustrando apenas ramificações para inferências quanto aos padrões de similaridade. Entretanto, delimitações para formação de determinado número de grupos podem ser definidas com base em exames visuais ou técnicas estatísticas. Neste trabalho, a linha de corte para estabelecer o número de grupos, bem como os tratamentos pertencentes, foi determinada pelo número de grupos formados pelo método de Tocher. A distância em porcentagem de aproximadamente $22 \%$ foi o nível adotado para o ponto de corte, mantendo o mesmo número de partições entre os dois métodos. Os resultados validaram os métodos, demonstrando equivalência entre os agrupamentos, cujos grupos foram constituídos pelas mesmas densidades.

Nesta população, cuja característica sob seleção é de baixa herdabilidade, a utilização de um mapeamento fino (alta densidade) proporciona maior poder de detecção de QTL e, consequentemente, melhores incrementos fenotípicos ao longo das gerações sob SAM. Santos et al. (2006) relacionaram o efeito do QTL à herdabilidade da característica. Assim, a identificação de QTL de pequeno efeito requer grande quantidade de marcadores estrategicamente ordenados. Resultados comparáveis foram observados entre as densidades que admitiram distribuição agregada de um marcador a cada 2, 4, 6, 8, 10, 12 e 14 cM, maximizando os ganhos com a seleção genômica.

\section{Característica de média herdabilidade $\left(h^{2}=0,40\right)$}

A Tabela 3 apresenta os valores fenotípicos médios ( \pm desvio-padrão) obtidos ao longo das gerações sob SAM. Foram mantidos os padrões de espaçamento entre marcadores, conforme mencionado na característica anterior. Novamente, resultados comparáveis foram observados entre os mapeamentos que adotaram alta resolução, exceto para a densidade de $2 \mathrm{cM}$, cujos valores fenotípicos assemelhavam-se aos espaçamentos com aproximadamente 20 cM de distância. Maximização nos ganhos com a seleção genômica foi obtida para as densidades de 4, 6, 8 e 10 cM. Resultados expressivos também foram identificados nas seleções que utilizaram até 59 marcadores (16 cM). Contudo, os mapas de baixa densidade (26, 28 e $30 \mathrm{cM}$ ) mantiveram os piores resultados ao longo das gerações, pois o baixo refinamento diminui o poder de deteç̧ão de QTL.

Ao utilizar uma distribuição muito saturada em marcadores espera-se grande poder de detecção. Entretanto, na prática essa alta densidade em algumas regiões genômicas poderá ter efeito contrário. Marcadores muito próximos poderão apresentar uma junção em seus efeitos, ocasionando redução na capacidade de identificar associações entre marcadores e QTL. Dois ou mais marcadores minimamente espaçados poderão funcionar como um único (Schuster \& Cruz, 2004). Dessa forma, a redução excessiva no espaçamento entre marcadores pode não resultar em ganhos apreciáveis na seleção genômica, conforme relatado para a densidade de $2 \mathrm{cM}$.

Procedeu-se a análise de agrupamento utilizando os valores fenotípicos médios obtidos ao longo das 20 gera-

Tabela 4 . Grupos, limite e distâncias obtidas entre os tratamentos (densidades de marcadores) pela aplicação do método de Tocher para herdabilidade de 0,40

\begin{tabular}{|c|c|c|c|c|}
\hline Número do grupo & Limite (è) & $\begin{array}{l}\text { Distância } \\
\text { obtida }\end{array}$ & $\begin{array}{l}\text { Número de } \\
\text { tratamentos }\end{array}$ & $\begin{array}{l}\text { Tratamentos } \\
\text { pertencentes }\end{array}$ \\
\hline 1 & 0,4875 & 0,14 & 2 & $10 ; 11$ \\
\hline 1 & 0,4875 & 0,17 & 3 & $10 ; 11 ; 12$ \\
\hline 1 & 0,4875 & 0,22 & 4 & $10 ; 11 ; 12 ; 1$ \\
\hline 1 & 0,4875 & 0,25 & 5 & $10 ; 11 ; 12 ; 1 ; 9$ \\
\hline 2 & 0,4875 & 0,17 & 2 & $14 ; 15$ \\
\hline 3 & 0,4875 & 0,17 & 2 & $7 ; 8$ \\
\hline 3 & 0,4875 & 0,23 & 3 & $7 ; 8 ; 6$ \\
\hline 4 & 0,4875 & 0,18 & 2 & $2 ; 5$ \\
\hline 4 & 0,4875 & 0,30 & 3 & $2 ; 5 ; 4$ \\
\hline 4 & 0,4875 & 0,47 & 4 & $2 ; 5 ; 4 ; 3$ \\
\hline 5 & 0,4875 & $* * * *$ & 1 & 13 \\
\hline
\end{tabular}




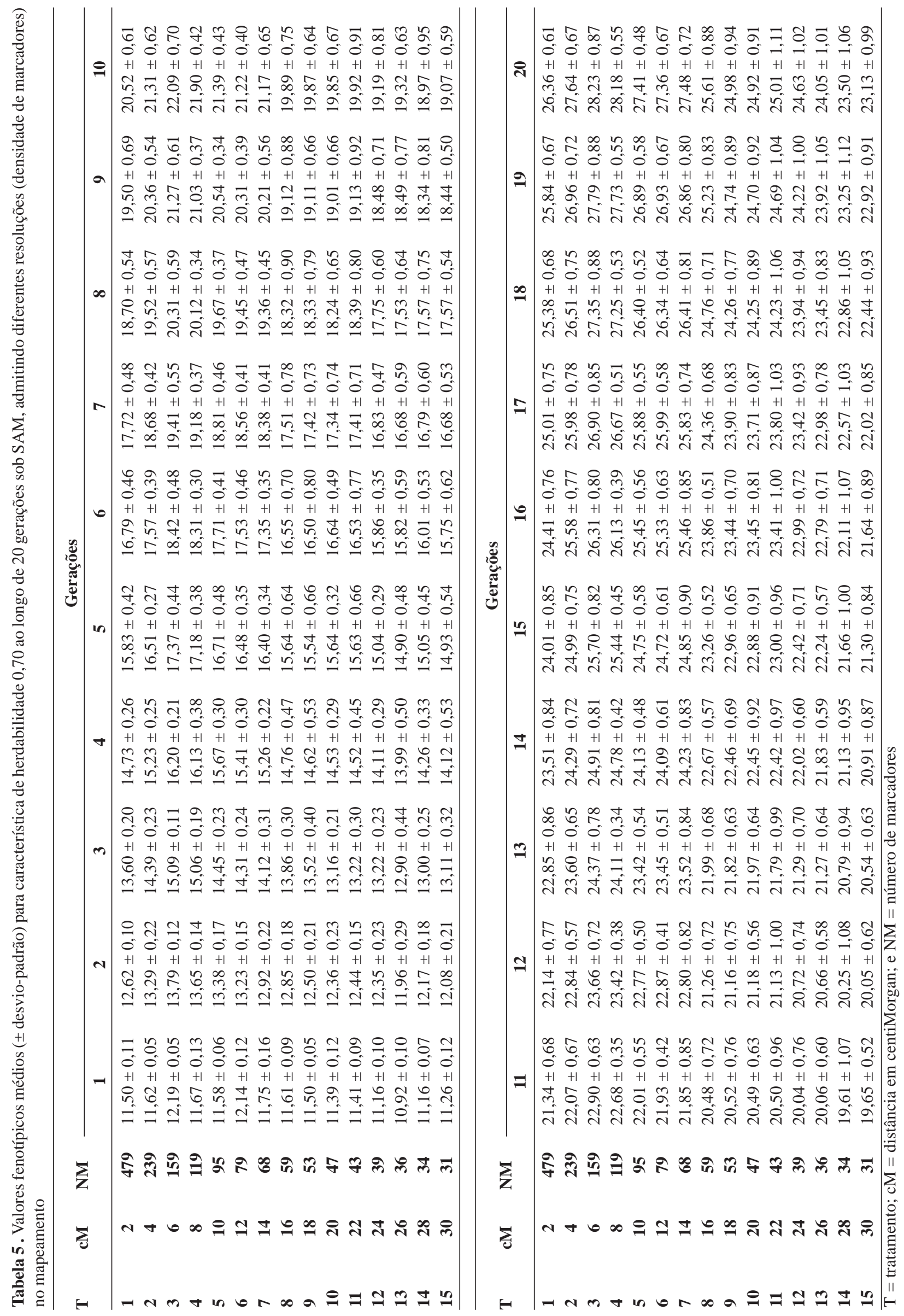

Rev. Ceres, Viçosa, v. 57, n.6, p. 695-700, nov/dez, 2010 
ções. O método de Tocher indicou a formação de cinco grupos, conforme a Tabela 4. No grupo 1 foram classificados os espaçamentos de 2, 18, 20, 22 e 24 cM. O grupo 2 inseriu os de 28 e $30 \mathrm{cM}$. O terceiro grupo foi formado pelas densidades de 12, 14 e 16 cM. As distâncias de 4, 6, 8 e 10 cM agruparam-se no quarto grupo. Já o grupo 5 compreendeu apenas a saturação que usou um marcador a cada 26 cM. Evidencia-se, com base nos incrementos fenotípicos médios, a inferioridade dos grupos 2 e 5 e a superioridade dos grupos 3 e 4 . Ganhos fenotípicos intermediários foram obtidos pelas densidades que constituíram o grupo 1 .

A Figura 2 ilustra o dendrograma estabelecido pelo método da ligação completa. Mantendo a distância de aproximadamente $22 \%$ como ponto de corte, tem-se a formação do mesmo número de grupos propostos pelo método de Tocher. Equivalência entre as densidades que constituíram os grupos foi observada nos dois métodos.

Nessa característica de herança genética 0,40 , o refinamento no mapeamento está relativamente associado à maximização dos ganhos com a seleção genômica. A otimização do poder de detecção de QTL não está proporcionalmente relacionada às maiores saturações. O maior vínculo genético do caráter favorece uma distribuição menos agregada de marcadores, permitindo menor gasto em material e mão de obra, além do ganho de tempo em prol de incrementos superiores. Maiores valores fenotípicos foram observados ao admitir as densidades de 4, 6, 8, $10,12,14$ e 16 cM.

\section{Característica de alta herdabilidade $\left(h^{2}=0,70\right)$}

Os valores fenotípicos médios ( \pm desvio padrão) obtidos na SAM, considerando característica de alta herdabilidade, são apresentados na Tabela 5. Valores fenotípicos superiores e correspondentes foram observados nas seleções que adotaram espaçamentos entre 4 e 14 cM ao longo de todas as gerações. Até as gerações intermediárias, a maior saturação (2 cM) indicou ganhos fenotípicos semelhantes aos espaçamentos entre 16 e 22 cM. Das gerações intermediárias até o final, essa alta resolução diferenciou-se das demais, proporcionando incrementos medianos. Otimização nos progressos foi evidenciada para as densidades de 6 e 8 cM. Todavia, os mapas de baixa densidade (26, 28 e $30 \mathrm{cM}$ ) mantiveram os piores resultados ao longo das gerações.

Os valores fenotípicos médios (Tabela 5) foram submetidos à análise de agrupamento. O método de Tocher indicou novamente a formação de cinco grupos, conforme a Tabela 6. No grupo 1 foram classificados os espaçamentos de 16, 18, 20, 22 e 24 cM. O grupo 2 inseriu os de 26, 28 e $30 \mathrm{cM}$. O terceiro grupo foi formado pelas densidades de 4, 10, 12 e 14 cM. As distâncias de 6 e 8 cM agruparam-se no quarto grupo. Já o grupo 5 compreendeu apenas a densidade de 2 cM. Evidenciase, com base nos incrementos fenotípicos, a inferioridade do grupo 2 e a superioridade dos grupos 3 e 4 . Ganhos fenotípicos intermediários foram obtidos pela den-

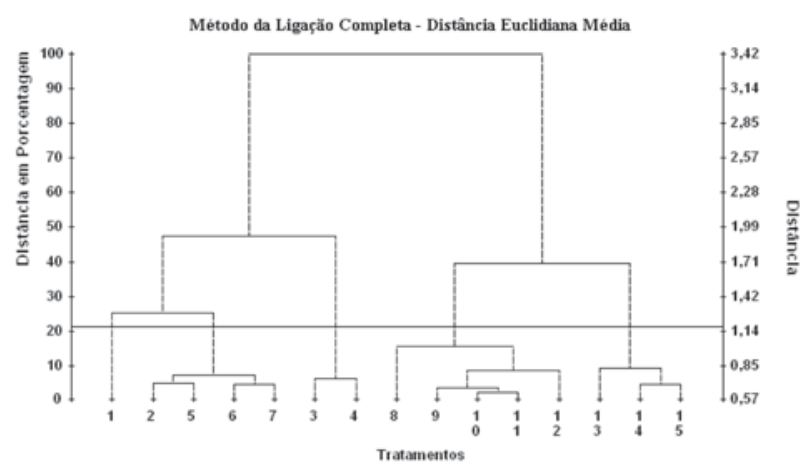

Figura 3 . Dendrograma do método da ligação completa pela análise de 15 tratamentos (densidades de marcadores) para herdabilidade de 0,70

Tabela 6 . Grupos, limite e distâncias obtidas entre os tratamentos (densidades de marcadores) pela aplicação do método de Tocher para herdabilidade de 0,70

\begin{tabular}{lcccc}
\hline Número do grupo & Limite $(\grave{e})$ & $\begin{array}{c}\text { Distância } \\
\text { obtida }\end{array}$ & $\begin{array}{c}\text { Número de } \\
\text { tratamentos }\end{array}$ & $\begin{array}{c}\text { Tratamentos } \\
\text { pertencentes }\end{array}$ \\
\hline $\mathbf{1}$ & 0,5703 & 0,08 & 2 & $10 ; 11$ \\
$\mathbf{1}$ & 0,5703 & 0,10 & 3 & $10 ; 11 ; 9$ \\
$\mathbf{1}$ & 0,5703 & 0,27 & 4 & $10 ; 11 ; 9 ; 12$ \\
$\mathbf{1}$ & 0,5703 & 0,38 & 5 & $10 ; 11 ; 9 ; 12 ; 8$ \\
$\mathbf{2}$ & 0,5703 & 0,15 & 2 & $14 ; 15$ \\
$\mathbf{2}$ & 0,5703 & 0,26 & 3 & $14 ; 15 ; 13$ \\
$\mathbf{3}$ & 0,5703 & 0,16 & 2 & $6 ; 7$ \\
$\mathbf{3}$ & 0,5703 & 0,18 & 3 & $6 ; 7 ; 2$ \\
$\mathbf{3}$ & 0,5703 & 0,20 & 4 & $6 ; 7 ; 2 ; 5$ \\
$\mathbf{4}$ & 0,5703 & 0,21 & 2 & $3 ; 4$ \\
$\mathbf{5}$ & 0,5703 & $* * * *$ & 1 & 1 \\
\hline
\end{tabular}

* Tratamentos: 1 (2 cM); 2 (4 cM); 3 (6 cM); 4 (8 cM); 5 (10 cM); 6 (12 cM); 7 (14 cM); 8 (16 cM); 9 (18 cM); 10 (20 cM); 11 (22 cM); 12 (24 cM); 13 (26 cM); 14 (28 cM); e 15 (30 cM) 
sidade de 2 cM. O método da ligação completa estabeleceu o dendrograma (Figura 3). O nível de corte foi instituído na distância próxima a 22\% para designar o mesmo número de grupos determinado pelo método de Tocher. Novamente, identidade entre os espaçamentos que constituíram os grupos foi observada para os dois métodos avaliados.

Neste cenário (característica de herdabilidade 0,70), o mapeamento saturado não maximizou os ganhos fenotípicos na seleção genômica. Infere-se que a otimização do poder de detecção de QTL não está associada às maiores resoluções. A elevada relação entre o genótipo e o fenótipo demanda uma distribuição menos densa de marcadores, pois a magnitude dos efeitos dos QTL detectados coincide com a herdabilidade da característica avaliada. Maiores incrementos fenotípicos foram observados ao admitir as densidades de 4, 6, 8, 10, 12 e $14 \mathrm{cM}$.

A Tabela 7 apresenta uma analogia dos padrões de agrupamento obtidos pela aplicação dos métodos da ligação completa e de Tocher para as diferentes herdabilidades. Evidenciou-se maior semelhança na formação de grupos entre as herdabilidades 0,40 e 0,70 ; em contrapartida, padrões desiguais de agrupamento foram obtidos ao relacionar as herdabilidades 0,10 e 0,40 .

Tabela 7 . Grupos com os tratamentos pertencentes pela aplicação dos métodos de agrupamento (Ligação Completa e Tocher), de acordo com a herdabilidade da característica

\begin{tabular}{lccc}
\hline Grupos & Tratamentos $^{*}\left(\mathbf{h}^{\mathbf{2}} \mathbf{= 0 , 1 0}\right)$ & Tratamentos $^{*} \mathbf{( \mathbf { h } ^ { 2 } = \mathbf { 0 , 4 0 } )}$ & Tratamentos $^{*}\left(\mathbf{h}^{2}=\mathbf{0 , 7 0}\right)$ \\
\hline $\mathbf{1}$ & $13 ; 14 ; 15$ & $1 ; 9 ; 10 ; 11 ; 12$ & $8 ; 9 ; 10 ; 11 ; 12$ \\
$\mathbf{2}$ & $8 ; 9 ; 10$ & $14 ; 15$ & $13 ; 14 ; 15$ \\
$\mathbf{3}$ & $1 ; 4 ; 5 ; 6 ; 7$ & $6 ; 7 ; 8$ & $2 ; 5 ; 6 ; 7$ \\
$\mathbf{4}$ & $11 ; 12$ & $2 ; 3 ; 4 ; 5$ & $3 ; 4$ \\
$\mathbf{5}$ & $2 ; 3$ & 13 & 1 \\
\hline${ }^{*}$ Tratamentos: 1 (2 cM); 2 (4 cM); 3 (6 cM); $4(8 \mathrm{cM}) ; 5(10 \mathrm{cM}) ; 6(12 \mathrm{cM}) ; 7(14 \mathrm{cM}) ; 8(16 \mathrm{cM}) ; 9(18 \mathrm{cM}) ; 10(20 \mathrm{cM}) ; 11(22 \mathrm{cM}) ;$ \\
$12(24 \mathrm{cM}) ; 13(26 \mathrm{cM}) ; 14(28 \mathrm{cM}) ;$ e $15(30 \mathrm{cM})$
\end{tabular}

12 (24 cM); 13 (26 cM); $14(28 \mathrm{cM}) ;$ e 15 (30 cM)

\section{CONCLUSÕES}

A análise de agrupamento indicou que as densidades de 4 e 6 cM; 4, 6, 8 e 10 cM; e 6 e 8 cM evidenciaram equivalência na detecção de QTL, otimizando os ganhos fenotípicos para as características de baixa, média e alta herdabilidades, respectivamente. Os métodos de Tocher e da ligação completa proporcionaram partições entre as densidades de marcadores iguais, implementando e validando as inferências a cerca das estruturas de classificação.

\section{REFERÊNCIAS}

Ambo M, Campos RLR, Moura ASMT, Boschiero C, Rosário MF, Ledur MC, Nones K \& Coutinho LL (2008) Genetic linkage maps of chicken chromosomes 6, 7, 8, 11 and 13 from a brazilian resource population. Scientia Agricola, 65:447-452.

Carneiro PLS, Machado CHM, Euclydes RF, Carneiro APS \& Cunha EE (2007) Endogamia, fixação de alelos e limite de seleção em populações selecionadas por métodos tradicionais e associados a marcadores moleculares. Revista Brasileira de Zootecnia, 36:369-375.

Darvasi A, Weinreb A, Minke V, Weller JI \& Soller M (1993) Detecting marker-QTL linkage and estimating QTL gene effect and map location using a saturated genetic map. Genetics, 134:943-951.

Euclydes RF (2009) Genesys: Sistema de Simulação Genética. Viçosa, Fundação Arthur Bernardes - UFV.

Ferreira DF (2008) Estatística Multivariada. Lavras, Editora UFLA. 662p.
Hillel J (1997) Map-based quantitative trait loci identification. Poultry Science, 76:1115-1120.

Ledur MC, Bertani GR \& Nones K (2003) Genômica nos programas de melhoramento genético avícola. In: Conferência Apinco de Ciência e Tecnologia Avícola, Campinas. Anais, APINCO. p.87-105.

Ledur MC, Nones K, Alves HJ \& Bertani GR (2004) A importância do conhecimento do genoma para a produção de aves. In: $41^{\text {a }}$ Reunião Anual da Sociedade Brasileira de Zootecnia, Campo Grande. Anais. p.379-389.

Ledur MC \& Schmit GS (2000) Genética Molecular: Aplicação de tecnologias moleculares no melhoramento genético de aves. Revista Avicultura Industrial, 90:13-19.

Malone G, Marchioro VS, Zimmer PD, Malone E, Carvalho FIF \& Oliveira AC (2007) Efeito do método de condução de populações sobre a similaridade genética de uma população segregante de aveia para peso de panícula. Scientia Agraria, 8:75-80.

Moreau L, Charcosset A, Hospital F \& Gallais A (1998) Markerassisted selection efficiency in populations of finite size. Genetics, 148:1353-1365.

Nascimento Filho FJ, Atroch AL, Sousa NR, Garcia TB, Cravo MS \& Coutinho EF (2001) Divergência genética entre clones de guaranazeiro. Pesquisa Agropecuária Brasileira, 36:501-506.

Neves HHR, Cavalheiro R, Cardoso V, Fries LA \& Queiroz AS (2009) Acasalamento dirigido para aumentar a produção de animais geneticamente superiores e reduzir a variabilidade da progênie em bovinos. Revista Brasileira de Zootecnia, 38:1201-1204.

Peixoto MGCD, Martinez ML, Teodoro RL, Machado MA, Carvalho MRS, Gomes FC \& Verneque RS (2009) Detecção de QTL em dados de famílias estruturadas como as de um núcleo MOET por meio do método da regressão. Arquivo Brasileiro de Medicina Veterinária e Zootecnia, 61:941-948.

Rev. Ceres, Viçosa, v. 57, n.6, p. 695-700, nov/dez, 2010 
Ribeiro Junior JI \& Melo ALP (2008) Guia prático para utilização do SAEG. Viçosa, Editora UFV. 288p.

Rodrigues JIS, Miranda FD, Ferreira A, Borges LL, Ferreira MFS, Good-God PIV, Piovesan ND, Barros EG, Cruz CD \& Moreira MA (2010) Mapeamento de QTL para conteúdos de proteína e óleo em soja. Pesquisa Agropecuária Brasileira, 45:472-480.

Rosa GJM (2007) Delineamento de experimentos em genética genômica. Revista Brasileira de Zootecnia, 36:211-218.

Ruy DC, Nones K, Baron EE, Ledur MC, Melo CMR, Ambo M, Campos RLR \& Coutinho LL (2005) Strategic marker selection to detect quantitative trait loci in chicken. Scientia Agricola, 62:111-116.

Santos MA, Nicolás MF \& Hungria M (2006) Identificação de QTL associados à simbiose entre Bradyrhizobium japonicum, B. elkanii e soja. Pesquisa Agropecuária Brasileira, 41:67-75.
Schuster I \& Cruz CD (2004) Estatística genômica aplicada a populações derivadas de cruzamentos controlados. Viçosa, Editora UFV. 568p.

Silva MVGB, Martinez ML, Torres RA, Lopes PS, Euclydes RF, Pereira CS, Machado MA \& Arbex W (2005) Modelos aleatórios na estimação da localização de QTLs em famílias de meiosirmãos. Revista Brasileira de Zootecnia, 34:66-75.

Vieira EA, Nodari RO, Carvalho FIF \& Fialho JF (2006) Mapeamento genético de caracteres quantitativos e sua interação com o ambiente. Planaltina, Documentos / Embrapa Cerrados. 28p. 